\title{
Cardiac Tamponade: An Unusual Cause of a Severe Headache with Normal Blood Pressure
}

\author{
Phool Iqbal ${ }^{1}$, Safna Farsana Akkam Veettil ${ }^{1}$, Arwa Alsaud ${ }^{1}$, Nagham Sadik ${ }^{1}$, Sania Razzaq ${ }^{2}$ \\ 1. Internal Medicine, Hamad General Hospital, Doha, QAT 2. Radiology, Hamad General Hospital, Doha, QAT
}

Corresponding author: Phool Iqbal, dr.phooliqbal@gmail.com

\begin{abstract}
Cardiac tamponade is a life-threatening condition characterized by slow or rapid compression of the heart due to the accumulation of fluid in the pericardial space and rarely presents mainly as a headache.

We report an unusual presentation of cardiac tamponade associated mainly with severe headache over three days and mild shortness of breath in a 60 -year-old male not known to have any previous heart disease. Immediate computed tomography (CT) scan of the head ruled out intracranial hemorrhage. A chest $\mathrm{x}$-ray showed cardiomegaly, and further echocardiography revealed a large pericardial effusion on a transthoracic echocardiogram compromising the right ventricular output. Urgent pericardiocentesis was performed and removed $800 \mathrm{ml}$ of hemorrhagic fluid that led to a complete resolution of his severe headache.
\end{abstract}

Received 02/04/2020

Review began 02/09/2020 Review ended 02/12/2020 Published 02/15/2020

() Copyright 2020 Iqbal et al. This is an open access article distributed under the terms of the Creative Commons Attribution License CC-BY 4.0., which permits unrestricted use, distribution, and reproduction in any medium, provided the original author and source are credited.
Categories: Cardiology, Internal Medicine, Radiology

Keywords: cardiac tamponade, normal blood pressure, headache

\section{Introduction}

Cardiac tamponade is a clinical syndrome caused by the accumulation of fluid, gas, pus, or blood in the pericardial space, resulting in reduced diastolic ventricular filling and subsequent hemodynamic compromise [1]. The condition is a medical emergency that can lead to cardiac arrest and death if not treated in a timely manner [2].

Headache as the main presentation of a potentially fatal condition like cardiac tamponade without any underlying secondary cause is quite an uncommon finding in the literature. Herein, we are reporting the case of a 60 -year-old male with an unusual presentation of cardiac tamponade.

\section{Case Presentation}

A 60 -year-old morbidly obese gentleman, a chronic smoker and alcohol drinker with a medical history significant for uncontrolled hypertension, type 2 diabetes mellitus with microvascular complications, stroke without residual weakness, and obstructive sleep apnea, presented to the emergency department with a three-day history of progressive severe headache. He described it as the worst headache of his life, involving the whole cranium and associated with mild blurriness of vision but without photophobia or any other focal neurological deficit.

He had cervical and lumbar spine decompression surgery and a renal transplant 11 years earlier, as well as stable renal graft function on immunosuppressant medications (prednisolone, tacrolimus, and mycophenolate).

On physical examination, the patient was having mild distress in a sitting position but was hemodynamically stable, afebrile with normal blood pressure, a pulse rate of $98 / \mathrm{min}$, and a respiratory rate of $24 / \mathrm{min}$ on $3 \mathrm{~L} / \mathrm{min}$ nasal cannula maintaining oxygen saturation of $97 \%$. His body mass index (BMI) was $42 \mathrm{~kg} / \mathrm{m}^{2}$. He had mild bilateral pedal edema. We were unable to comment on jugular venous pulsations and heart sounds due to his thick short neck and chest wall, respectively. Neurological examination was unremarkable for any focal motor or sensory deficit and cranial nerve examination was normal. Fundoscopic examination of the optic disk was unremarkable for any papilledema.

An urgent computed tomography (CT) scan brain ruled out subarachnoid hemorrhage as shown in Figure 1. Magnetic resonance imaging (MRI) of the brain with contrast showed generalized volume loss of the brain as shown in Figure 2. Lumbar puncture was difficult to perform due to the history of metallic fixators in the lumbar spine region. 


\section{Cureus}

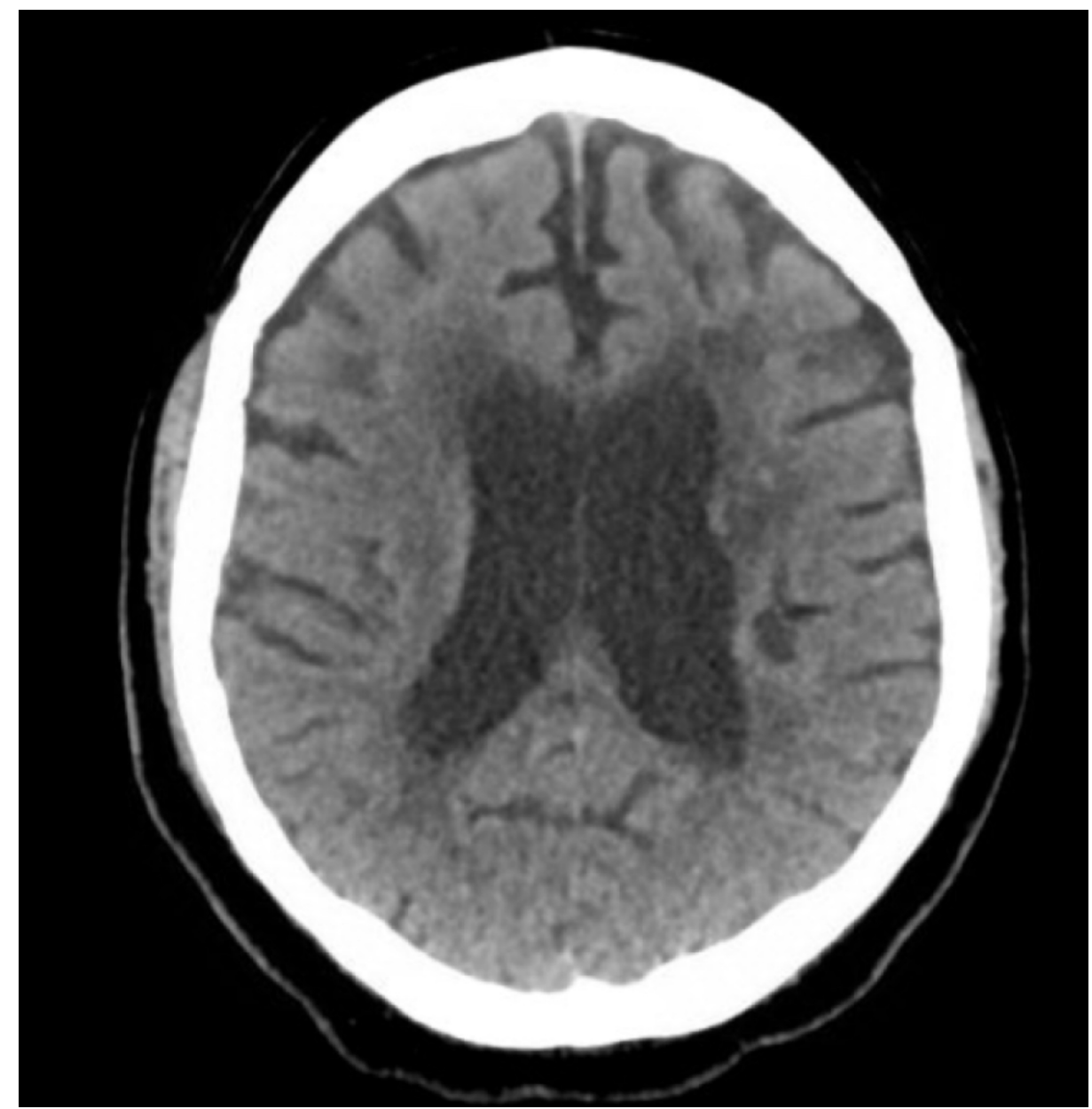

FIGURE 1: Computed tomography scan brain unremarkable for subarachnoid hemorrhage or any other acute insult 


\section{Cureus}

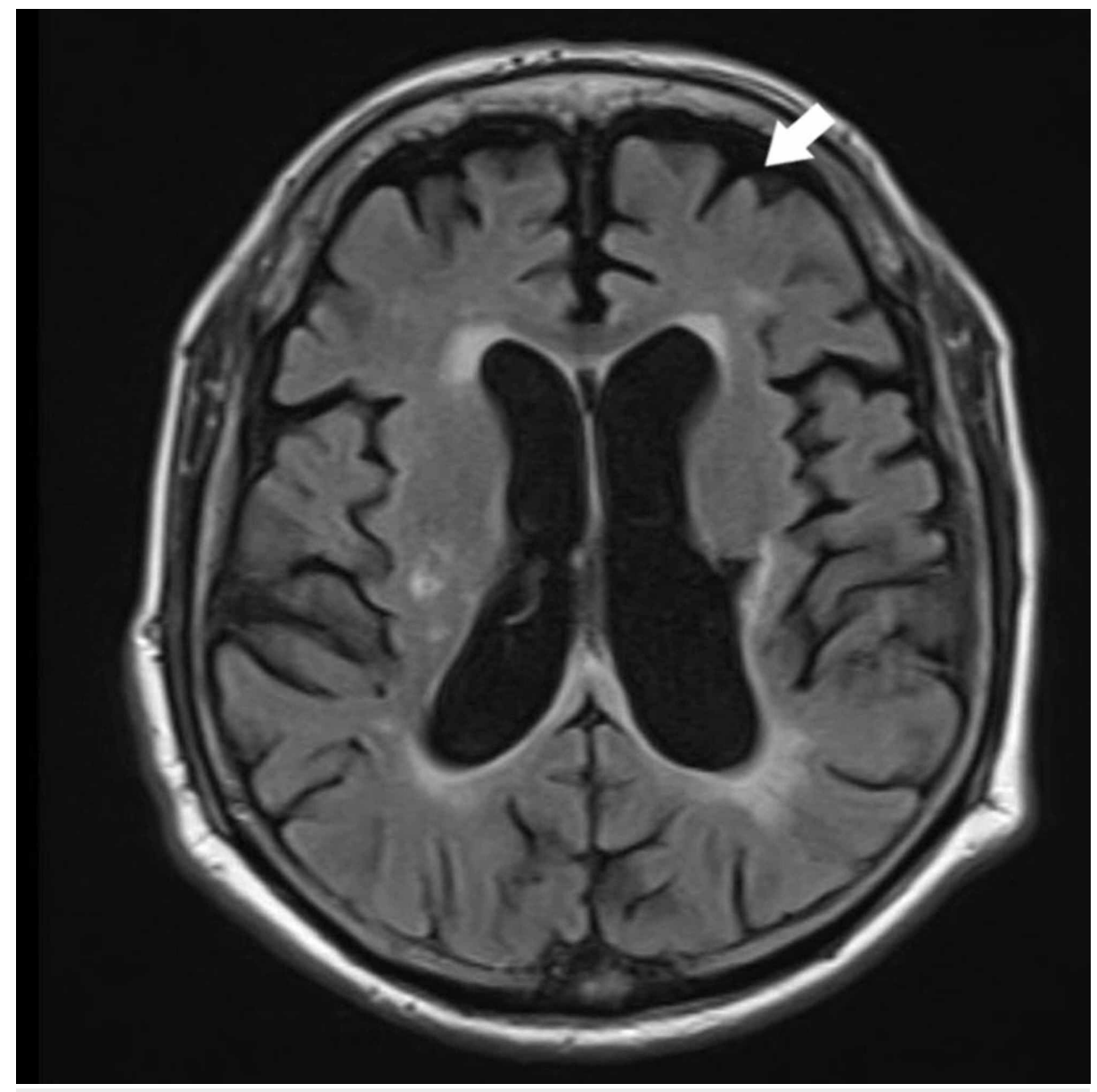

FIGURE 2: Magnetic resonance imaging (MRI) of the brain

The arrow points towards sulcal widening and gyri atrophy correlating with generalized brain volume loss and atrophy

An electrocardiogram (ECG) showed low voltage sinus rhythm and no beat-to-beat variation. Chest x-ray showed mild left pleural effusion and cardiomegaly as seen in Figure 3. Echocardiography was performed which revealed a large pericardial effusion with features of tamponade that included a large circumferential pericardial effusion with respiratory variation greater than $25 \%$ and right ventricular compression during systole and diastole. 


\section{Cureus}

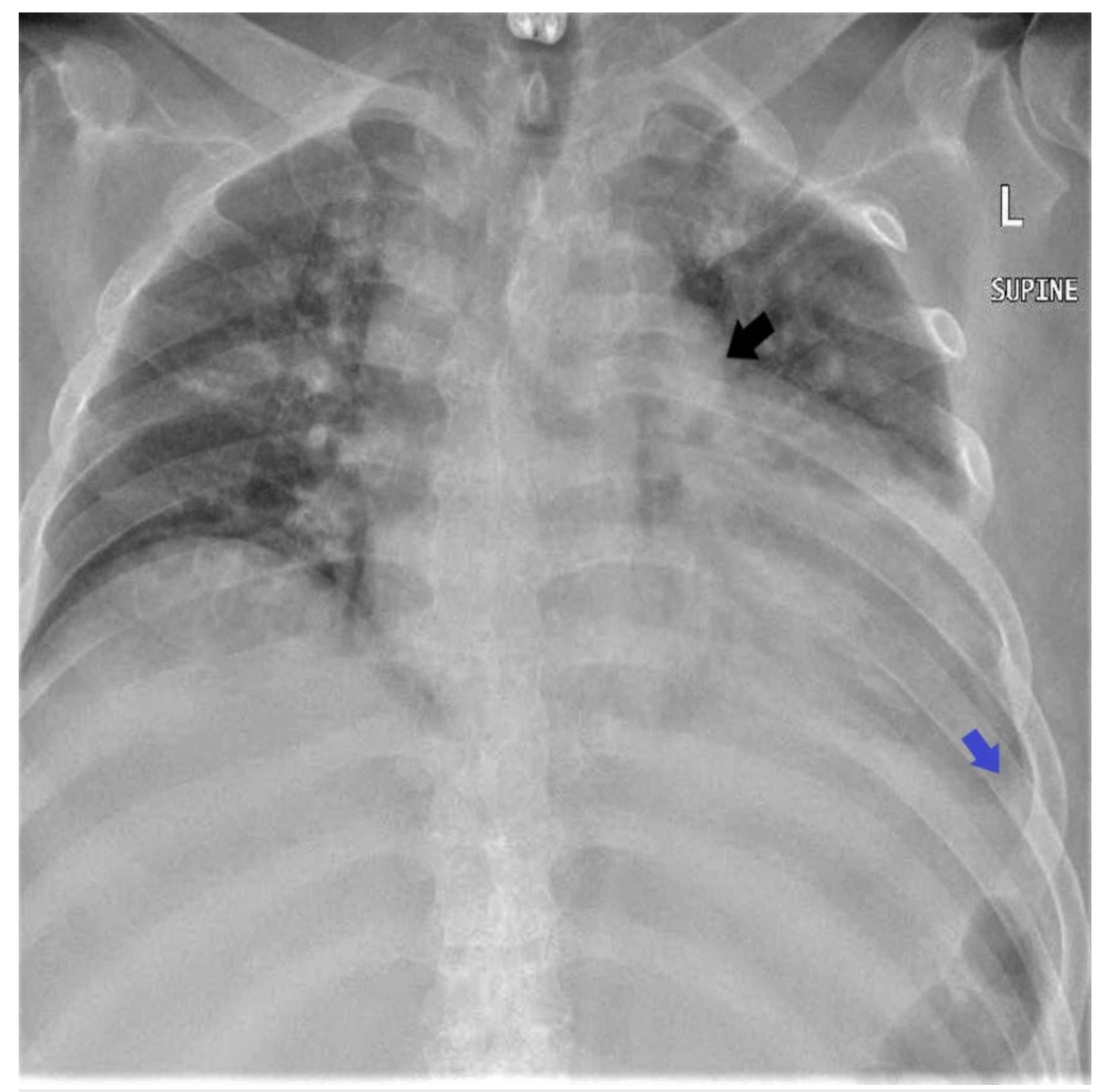

FIGURE 3: X-ray revealing a large pericardial effusion with features of tamponade

The black arrow points towards the increased cardiac silhouette suggestive of pericardial effusion; the blue arrow shows a left blunted costophrenic angle due to pleural effusion.

The patient was taken to the coronary care unit and underwent emergent pericardial drainage as described in Figure 4. Approximately $800 \mathrm{~mL}$ of hemorrhagic pericardial fluid was drained. The fluid analysis showed 750 /uL white blood cells with mainly $74 \%$ neutrophilic, $1 \%$ lymphocytes and $50,750 \mathrm{RBC} / \mathrm{uL}$ concluding it as a hemorrhagic pericardial effusion. Acid-fast bacilli (AFB) smear, tuberculosis (TB) polymerase chain reaction (PCR), fluid culture, and viral markers were negative from the pericardial fluid analysis. Vasculitis screening with antinuclear antibody (ANA) and antineutrophil cytoplasmic antibody (ANCA) was negative as well. The septic screen was also sent for the patient and his blood cultures did not grow any microorganism.
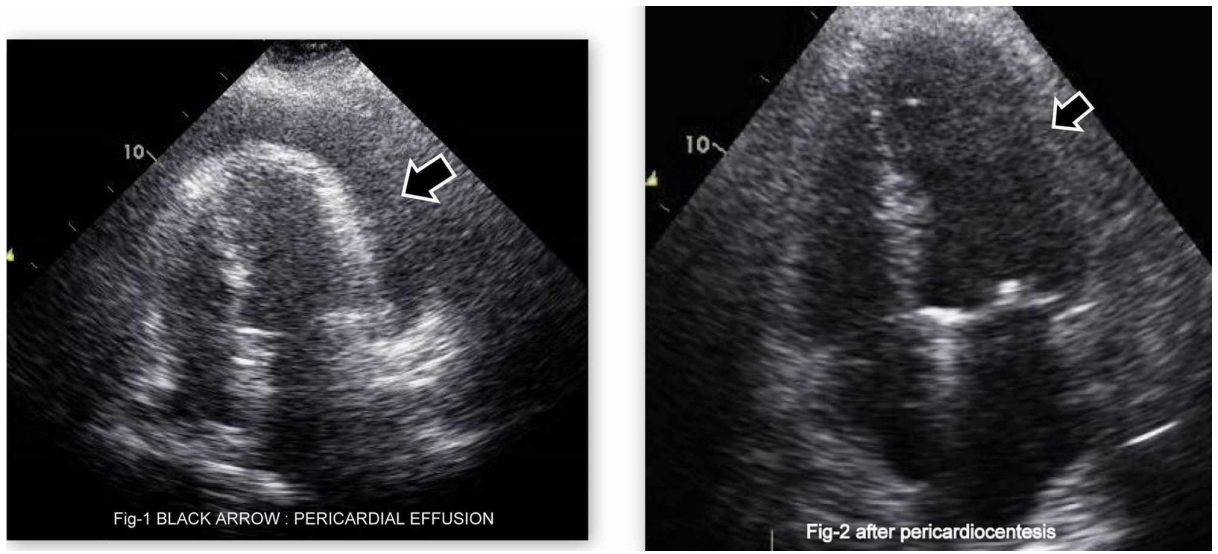

FIGURE 4: Before (left side) and after (right side) pericardiocentesis 


\section{Cureus}

\section{echocardiography of the patient}

The patient's headache was relieved after pericardiocentesis. His headache did not recur during his remaining length of hospital stay. However, his blood pressure started to shoot up to $180-220 \mathrm{mmHg}$ systolic over $90-120 \mathrm{mmHg}$ diastolic for which we resumed his home antihypertensive medications and it stabilized.

A positron emission tomography/computed tomography (PET CT) scan did not detect any malignant focus with enhanced uptake. An MRI scan of the heart showed basal septal mid-wall myocardial enhancement of non-ischemic pattern, likely due to myocarditis or cardiomyopathy as shown in Figure 5.

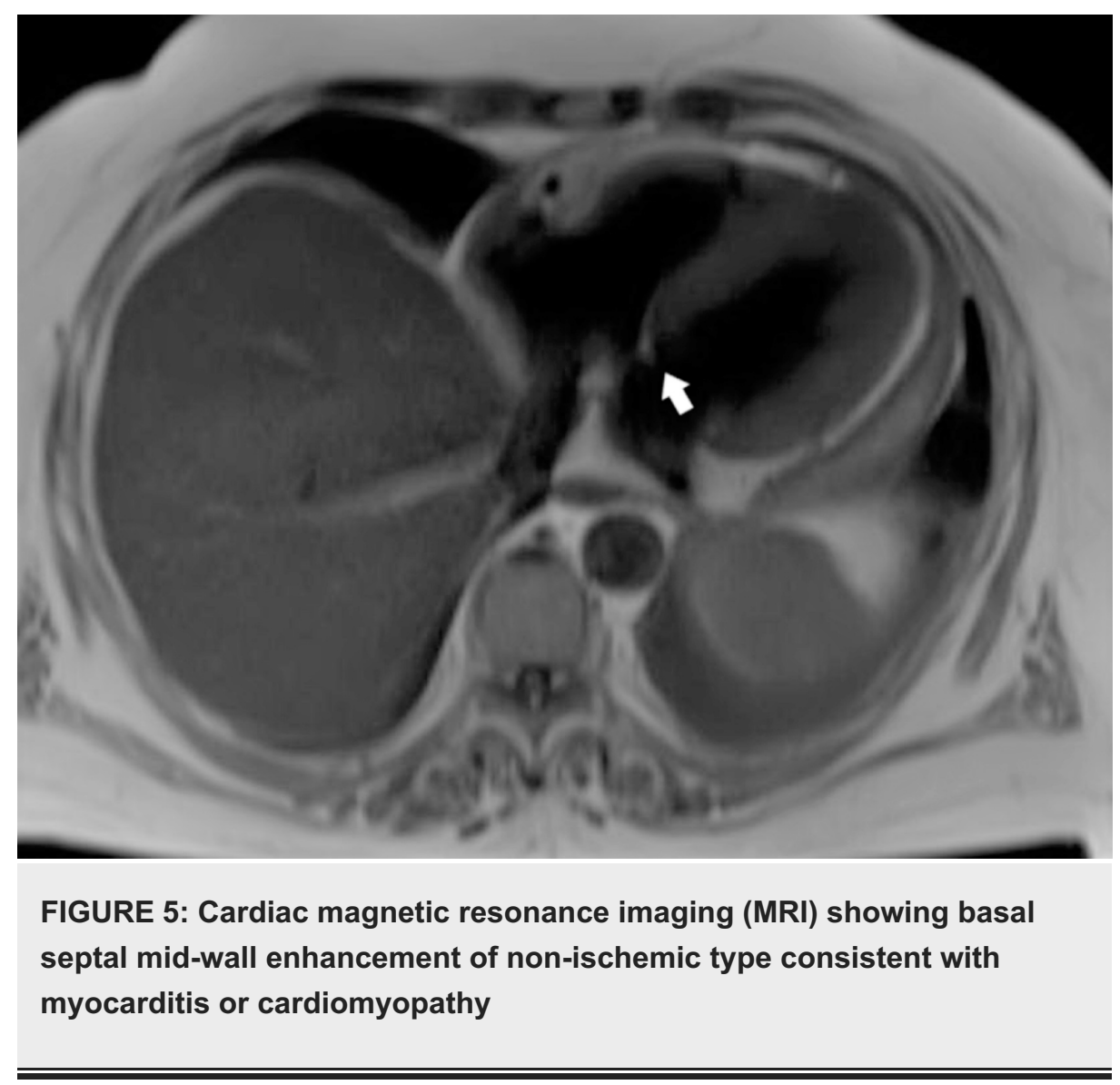

After ruling out secondary causes of hemorrhagic pericardial effusion, such as malignancy, autoimmune diseases, and TB, our patient was labeled as idiopathic myocarditis leading to chronic pericardial effusion based on MRI scan of the heart. We observed the patient in the hospital with a regular echocardiogram over 10 days. He was asymptomatic and discharged with regular follow-up in the cardiology clinic. Cardiologist outpatient follow-ups with repeated echocardiogram studies for a further five months did not show any fluid reaccumulation. The patient was followed for one year after discharge where he remained clinically stable.

\section{Discussion}

The heart is enclosed in the pericardium which is a double-layered sac. Pericardial fluid is a lubricating substance in between the two layers of the pericardium and is approximately $50 \mathrm{ml}$ in quantity. Its main function is to reduce the friction between the pericardial layers and provide antimicrobial properties [3].

The hemodynamics of the heart in cardiac tamponade is disrupted. It occurs when the pressure inside the heart chambers is insufficient to overcome the increased interpericardial pressure during diastole. The heart chambers also become smaller in size due to external pericardial pressure during the cardiac cycle, leading to a decreased venous return to the right side of the heart. Consequently, it compromises cardiac output leading to hypotension. However, tachycardia is seen as one of the early compensatory response mechanisms to maintain cardiac output [3-5]. Signs and symptoms of cardiac tamponade depend on the rate of accumulation, i.e., acute versus subacute or chronic, pericardial stretch, and ventricular compliance [3-4].

A classic presentation of cardiac tamponade includes Beck's triad which is hypotension, increased jugular venous pulse, and muffled heart sounds, while chest pain, dyspnea, tachycardia, pulsus paradoxus, and 
cardiovascular collapse can also occur. In acute settings, the patient can deteriorate within minutes even with minimal increase in pericardial fluid up to $150 \mathrm{ml} \mathrm{[3-5].}$

In subacute or chronic settings, the stretch of the pericardium is accommodative to slow rising interpericardial fluid. Therefore, it can retain even more than $1,000 \mathrm{ml}$ over days to months, and the patient may still be asymptomatic as long as diastolic pressure of the heart is more than pericardial pressure [3]. Once, the diastolic pressure is no longer sufficient enough to overcome the rising pericardial pressure, the patient becomes symptomatic and can present with fatigability, mild dyspnea, peripheral edema, and chest discomfort. Rare chronic cases can have a circulatory collapse [3-5].

There is a chronic compression triad similar to Beck's triad, and it is accompanied by high venous pressure, abdominal ascites, and muffled heart sounds [6].

Our patient did not present with typical features of cardiac tamponade and came primarily with the symptom of headache, which is rare to be seen in such life-threatening conditions with relatively normal blood pressure.

Headache due to cardiac tamponade can be explained by the inability of cerebral veins to drain into the superior vena cava and then into the right atrium due to external compression by massive pericardial effusion. As cerebral vessels are pain-sensitive structures, this leads to stretch on the cerebral vessels that trigger pain sensation and traction type of headache [7-8].

Headache in association with cardiac tamponade has been reported in few case reports, but there were underlying diseases, such as dengue fever, lupus nephritis, and superior vena cava syndrome, which explained the headache in those patients [9-11].

Our patient was known to have uncontrolled blood pressure and was on four different types of antihypertensive medications. However, due to the cardiac tamponade, he presented with normal blood pressure which created a dilemma to diagnose such scenarios. This emphasizes the importance of history taking and background in such patients.

He had mild shortness of breath, his heart sounds were difficult to detect due to thick chest walI, and jugular venous pulsations could not be appreciated due to a short neck. Therefore, the decision of echocardiography was made after his chest x-ray reported cardiomegaly and suspected pericardial effusion. This led to the diagnosis of massive pericardial effusion leading to tamponade. After urgent pericardiocentesis, the patient's headache and shortness of breath were relieved and his blood pressure started to shoot up which required us to resume his antihypertensive medication.

The pericardial fluid analysis revealed hemorrhagic pericardial effusion, and the patient underwent extensive investigations that ruled out secondary causes like tuberculosis, malignancy, and autoimmune diseases. Thus, it was determined to be a case of idiopathic myocarditis on basis of cardiac MRI, complicated by massive chronic hemorrhagic pericardial effusion and cardiac tamponade. Idiopathic myocarditis is a common cause of pericardial effusion found in the literature. We did a close follow-up for one year and the patient remained asymptomatic without any reaccumulation of pericardial effusion on repeat echocardiography as an outpatient. However, if there should be a recurrence of pericardial effusion and no other cause can be established from clinical and laboratory investigations, then a biopsy can help in such clinical scenarios [12]. Our patient did not require it.

Echocardiography is the best modality to look for pericardial effusion and is used as a tool in follow-up [2-3]. Our patient was followed with serial transthoracic echocardiography and he remained stable throughout our follow-up.

\section{Conclusions}

Cardiac tamponade is a life-threatening condition that can lead to cardiac arrest and death if not promptly managed. Our patient mainly presented with a severe intractable headache associated with this fatal condition which was relieved immediately after pericardiocentesis. Our main goal is to highlight the importance of history taking, physical examination, and approach towards this atypical presentation associated with cardiac tamponade and its urgent management for better patient care. As per the literature review, and to our knowledge, headache as the main presenting symptom of cardiac tamponade due to idiopathic myocarditis has not been reported.

\section{Additional Information \\ Disclosures}

Human subjects: Consent was obtained by all participants in this study. Conflicts of interest: In compliance with the ICMJE uniform disclosure form, all authors declare the following: Payment/services info: All authors have declared that no financial support was received from any organization for the 
submitted work. Financial relationships: All authors have declared that they have no financial relationships at present or within the previous three years with any organizations that might have an interest in the submitted work. Other relationships: All authors have declared that there are no other relationships or activities that could appear to have influenced the submitted work.

\section{References}

1. Ariyarajah V, Spodick DH: Cardiac tamponade revisited: a postmortem look at a cautionary case . Tex Heart Inst J. 2007, 34:347-51.

2. Stashko E, Meer JM: Cardiac Tamponade. StatPearls (Internet). StatPearls Publishing, Treasure Island (FL); 2019.

3. Jensen JK, Poulsen SH, Mølgaard H: Cardiac tamponade: a clinical challenge . e-Journal of Cardiology Practice. 2017, 15:17. Accessed: February 14, 2020: http://www.escardio.org/Journals/E-Journal-ofCardiology-Practice/Volume-15/Cardiac-tamponade-a-clinical-challenge.

4. Khandaker MH, Espinosa RE, Nishimura RA, Sinak LJ, Hayes SN, Melduni RM, Oh JK: Pericardial disease: diagnosis and management. Mayo Clin Proc. 2010, 85:572-93. 10.4065/mcp.2010.0046

5. Shabetai R: Pericardial effusion: haemodynamic spectrum . Heart. 2004, 90:255-56. 10.1136/hrt.2003.024810

6. Pacha HM, Soud M, Alraies MC: Beyond Beck's triad: a rare cause of cardiac tamponade and hemoptysis . Ochsner J. 2018, 18:271-73. 10.31486/toj.17.0081

7. Bigley GK: Headache. Clinical Methods: The History, Physical, and Laboratory Examinations, 3rd edition. Walker HK, Hall WD, Hurst JW (ed): Butterworths, Boston; 1990.

8. Bayot ML, Zabel MK: Neuroanatomy, Dural Venous Sinuses. StatPearls. StatPearls Publishing, Treasure Island (FL); 2019.

9. Biswas S, Kumar P, Tansir G, Biswas A: Case report. Cardiac tamponade in dengue hemorrhagic fever: an unusual manifestation of a common disease. Am J Trop Med Hyg. 2019, 101:448-450. 10.4269/ajtmh.19-0153

10. Bendwal S, Malviya K, Jatav OP, Malviya K: Cardiac tamponade presenting as early manifestation in dengue fever. J Assoc Physicians India. 2014, 62:257-59.

11. Kumar S, Iuga A, Jean R: Cardiac tamponade in a patient with dengue fever and lupus nephritis: a case report. J Intensive Care Med. 2010, 25:175-78. 10.1177/0885066609358955

12. Kabukcu M, Demircioglu F, Yanik E, Basarici I, Ersel F: Pericardial tamponade and large pericardial effusions: causal factors and efficacy of percutaneous catheter drainage in 50 patients. Tex Heart Inst J. 2004, 31:398-403. 